\title{
EFFICACY AND SAFETY OF PLATELET RICH PLASMA(PRP) INJECTION IN THE TREATMENT OF DUPUTYREN'S CONTRACTURE
}

\author{
Metin Terzibasioglu Aynur ${ }^{1}$, Coskun Celik Evrim¹, Ones Kadriye ${ }^{1}$ \\ ${ }^{1}$ University of Health Sciences, Istanbul Physical Medicine and Rehabilitation Research \\ and Training Hospital-Istanbul,Turkey
}

Introduction: Duputyren's contracture (DC) (palmar fibromatosis) is a slowly progressive connective tissue disorder that affects the palmar fascia.

Purpose:To evaluate the efficacy and safety of platelet rich plasma (PRP) injection in the treatment of Duputyren's contracture and to assess patient satisfaction with this new treatment approach.

Method: Platelet rich plasma (PRP) is an increased concentration of autologous platelets suspended in a small amount of plasma after centrifugation. Local anesthetic ( $1 \%$ lidokain ) was administered before PRP injection. PRP injection into the Duputyren contracture cord in divided aliquots performed with 30 Gauge mesotherapy needle. After injection mobilisation and massage applied to the palm and fingers by patient herself. Cords were allowed to be injected at a maximum of 3 treatments at minimum of 1 - week interval. Patient was reevaluated at 30 days after initial treatment.
Result: We applied PRP injection 3 times in 1 week apart. After the first injection she had full range of motion and had no residual contracture.VAS score was 0 after treatment. She had no numbness and tingling.Her grasp strength increased. After one month, her VAS score was still 0 (zero) and her 4th metacarpophalangeal (MCP), proximal interphalangeal and 5th metacarpophalangeal (MCP) proximal interphalangeal joint range of motion were still normal and there was no contracture.She has a high degree of patient satisfaction after one week and one month. We observe no adverse events after one month follow up.

Conclusion: In literature we have not seen PRP (platelet rich plasma) injection therapy for the treatment of DC. Platelet rich plasma (PRP) injection safely and effectively restored normal finger extension and contractures. In short -term it is safe and effective in our hands. Additional longterm studies of large numbers of patients are warranted to verify the effectiveness and recurrance rate with PRP treatment.

\footnotetext{
References

1.Maria A. Badalamante, PhD, Lawrence C. Hurst, MD. Efficacy and Safety of Injectable Mixed Collagenase Subtypes in the Treatment of Duputyren's Contracture. J Hand Surg Am. 2007 Jul-Aug;32(6):767-74.

2. Odinsson A, Brenne LE, Lurie TB, Finsen V. Dupuytren's Contracture. The Safety and Efficacy of Collagenase Treatment. J Hand Surg Asian Pac Vol. 2016 Jun;21(2):187-92

3. Hwee YK, Park D, Vinas M, Litts C, Friedman D. Outcome of Dupuytren Contractures After Collagenase Clostridium Histolyticum Injection: A Single-institution Experience. Ann Plast Surg. 2017 Aug;79(2):145-148.
} 